\title{
(In) Noten suchen - Das Josquin Research Project
}

Ein ernüchternder Gedanke: Im weit fortgeschrittenen dritten Jahrzehnt des Internetzeitalters liegen keine digitalen Ausgaben des westlichen Musikkanons vor, geschweige denn ein zuverlässiges Mittel zur elektronischen Suche. Zwar bietet das Internet eine große Bandbreite chronologisch vielfältiger Partituren im PDF- und MIDI-Format an. ${ }^{1}$ Tools wie Themefinder und RISM ermöglichen den Zugriff auf kurze melodische Auszüge aus einem recht umfangreichen Korpus, ${ }^{2}$ und Suchmaschinen auf Basis der Optical Musical Recognition (OMR) versprechen, große Datensätze abfragen zu können. ${ }^{3}$ Dennoch liefern diese Tools noch nicht den benötigten Zugang.

Um nur ein Beispiel zu nennen: Gegenwärtig gibt es keine Möglichkeit zu untersuchen, wie viele Melodien des 18. Jahrhunderts ihren rhythmischen und intervallischen Gehalt mit den ersten drei Noten des Hauptthemas von Mozarts Symphonie Nr. 40 in g-Moll gemeinsam haben. ${ }^{4}$ Es ist nicht möglich zu eruieren, wie häufig dieses Motiv in seiner ursprünglichen Gestalt, zwei Mal in Folge oder in leicht veränderten rhythmischen und melodischen Konfigurationen verwendet wurde, ohne tausende Partiturseiten von Hand zu durchsuchen. Da dies in den meisten Fällen unpraktisch oder gar unmöglich ist, besteht fast immer das Risiko, dass bei der Charakterisierung von Stücken oder Stilen schwerwiegende Fehler gemacht werden. Je größer der Wunsch ist, eine bestimmte Passage als einzig-

1 Die am häufigsten verwendeten Tools sind die Petrucci Music Library (https://imslp.org) und die Choral Public Domain Library (http://www3.cpdl.org/wiki/index.php/Main_Page). Eine weitere wertvolle Ressource ist The McGill Billboard Project: https://ddmal.music.mcgill.ca/research/The McGill_Billboard_Project_(Chord_Analysis_Dataset)/. Neuerdings bietet auch die Bayerische Staatsbibliothek München eine entsprechende Anwendung an: https://scoresearch.musiconn.de.

2 www.themefinder.org; und https://opac.rism.info/metaopac/start.do? View $=$ rism\&SearchType $=2 \&$ Language $=$ en.

3 An der McGill Universität laufen interessante Forschungen in dieser Richtung, darunter Ichiro Fujinagas »Single Interface for Music Score Searching and Analysis«-Projekt (https://simssa.ca). Siehe außerdem die von Rainer Typke entwickelte www.musipedia.org/. Derzeit müssen sich die meisten OMR-basierten Suchvorgänge auf unkorrigierte, fehlerhafte Daten mit einer Fehlerquote von ca. 5 bis $10 \%$ stützen. So nützlich diese Tools auch als grobe Recherchehilfen sein mögen, können sie nur selten zuverlässige Erkenntnisse liefern, nicht zuletzt, weil jeder Fehler aufgrund seines vertikalen (d. h. harmonischen) Zusammenhangs multipliziert wird.

4 Gemeint ist hier natürlich die Themeneröffnung in den Streichern. Mit Hilfe von www.peachnote.com kann man sehr grob sehen, wie häufig diese melodische - nicht rhythmische - Sequenz über einen großen Zeitraum erklingt. Allerdings gibt es keine Möglichkeit, die Suchergebnisse genau zu recherchieren, geschweige denn, sie nach Komponist, Genre etc. zu filtern. 
artig zu bewerten, desto wahrscheinlicher können entsprechende Gegenbeispiele beigebracht werden; und je deutlicher für die normative Qualität eines Stückes argumentiert werden soll, desto mehr läuft man Gefahr, dass diesem irrtümlicherweise ein repräsentativer Stellenwert für einen größeren Korpus zugeschrieben wird. Natürlich muss sich jede Wissenschaft mit diesen Problemen in einem gewissen Maße auseinandersetzen; die Vorstellung von totaler »Kontrolle« ist trügerisch und ab einem gewissen Grad unmöglich. Dennoch wäre es fahrlässig, dieses Problem zu ignorieren: Bei aller Überzeugung, dass sich Forschung an digitalen Repertorien lohnt, müssen wir konzedieren, dass bislang nur die Spitze des Eisbergs erschlossen worden ist.

Hier setzt das Josquin Research Project (JRP) an. Das 2010 gegründete JRP (https://josquin.stanford.edu) ist eine open-access-Datenbank, die es ermöglicht, einen zentralen Musikkorpus von ca. 1420 bis ca. 1520 auffindbar, durchsuchbar und hörbar zu machen. Jeder kann vollständige Partituren herunterladen, MP3Dateien anhören und lineare Aspekte von Musik abfragen, darunter melodische, intervallische sowie rhythmische Modelle und Schemata, die vollständig in der Datenbank erfasst sind; das JRP zeigt Suchergebnisse mit hervorgehobenen Übereinstimmungen wie in einer Google-Suche an. Eine Reihe von Analysewerkzeugen gewähren Einblick in die Beschaffenheit eines Werkes, in seine Form, in die Behandlung von Dissonanz, ermöglichen Aussagen zum Vorkommen von Quint- und Oktavparallelen in der Musik eines bestimmten Komponisten oder zur relativen Häufigkeit verschiedener rhythmischer Muster in einer bestimmten Gattung. Aus der Heterogenität einer Vielzahl von Editionen, die jeweils unterschiedlichen Richtlinien folgen, ergibt sich ein Problem: In welcher Beziehung stehen editorische Entscheidungen zur ursprünglichen Mensuralnotation bzgl. der Verbalkung und des Metrums? Sollte ein Schlag durch eine Viertelnote, eine halbe Note oder eine ganze Note repräsentiert werden? Das JRP erleichtert durch seine einheitliche editorische Praxis Vergleiche sowohl mit den Originalquellen als auch mit allen modernen Notenausgaben, die wir herangezogen haben.

Diese Werkzeuge haben schon jetzt einen Zugang zu einer bislang unerreichten Anzahl von Texten eröffnet. Damit verfügen wir über eine neue Art der »Big Data «-Analyse, durch die nicht nur Erkenntnisse über individuelle musikalische Aspekte, sondern über ganze Repertorien gewonnen werden können. Darüber hinaus erschließen sie neue Untersuchungsgebiete und einen differenzierteren Umgang mit einzelnen Werken. Im Folgenden soll der Ursprung und Hintergrund des Projekts dargestellt werden, um im Anschluss daran repräsentative Beispiele vorzustellen, wie die Analysetools des JRP neue Forschungsansätze erschlossen haben. Abschließend sollen einige neue Forschungsergebnisse vorgestellt werden. Obwohl die hier angesprochenen Themen zu einem bestimmten 
Grad spezifisch für die Musik des 15. Jahrhunderts sind, lassen sie sich größtenteils auf jedes annotierte Repertorium anwenden. Es mag erstaunen, dass es heute möglich ist, die Kompositionen Ockeghems und Josquins, aber nicht diejenigen Bachs, Beethovens oder Bergs zu durchsuchen und mit digitalen Mitteln zu analysieren. Sicherlich besteht für Tools dieser Art ein enormes Potential, sowohl alte als auch jüngere Musik zu erforschen.

\section{Die Geschichte des Projekts}

1998 begann ich mit dem Aufbau einer digitalen Sammlung von Renaissancemusik, indem ich Faksimiles und Partituren in das Notationsprogramm Finale mit Hilfe eines MIDI Keyboards eingab. Ich verfolgte dabei nicht das Ziel, einen beliebigen Korpus zu digitalisieren, sondern nutzte den Computer als ein Tool, um Stücke zu lernen und zu einem besseren Verständnis der Musik zu gelangen. Hierzu spielte ich polyphone Kompositionen Stimme für Stimme in Finale in Echtzeit ein; während ich aufeinanderfolgende Stimmen eingab, wurden zeitgleich die bereits transkribierten Stimmen abgespielt, sodass ich hörte, wie sich das Stück nach und nach zusammensetzte. 2007 umfasste meine persönliche Datenbank mehrere hundert Stücke, die ich für meine eigene Forschung sowie als Grundlage für wissenschaftliche und praktische Editionen nutzte. Zu dieser Zeit fehlten mir jedoch die Mittel, diese Partituren zu durchsuchen, geschweige denn sie digital vergleichen oder ihre musikalischen Parameter analysieren zu können. 2010 gründeten Craig Sapp und ich schließlich das JRP in Kooperation mit dem Center for Computer Assisted Research in the Humanities (CCARH), einer Organisation des Stanford Department of Music, die sich auf die digitale Kodierung von Musik spezialisiert hat; außerdem gründeten wir einen internationalen wissenschaftlichen Beirat. Mit Hilfe von Studenten vergrößerten wir unsere Notendatenbank enorm, beginnend mit den Werken, die Josquin zugeschrieben werden, bis hin zu Werken von Ockeghem, Pierre de la Rue und weiteren, darunter ein wachsender Korpus von anonym überlieferten Werken.

Zunächst wurde das Projekt aus Fördermitteln finanziert, die uns darin unterstützen sollten, ein bekanntes wissenschaftliches Problem zu lösen: Von den ca. 340 Werken, die Josquin in Quellen des 15. und 16. Jahrhunderts zugeschrieben werden, kann nur ein Bruchteil als authentisch gelten. ${ }^{5}$ Unser erstes Ziel war es, diese Werke zu digitalisieren, um die Grundlage für eine vergleichende Analyse zu schaffen. Im Rückgriff auf einen Grundlagentext von Joshua Rifkin nannten wir dieses Projekt das »Josquin Research Project« in Anlehnung an das Rembrandt

5 Bei der Förderung handelte es sich um einen 2010 Hellman Faculty Scholar Award. 
Research Project, welches sich mit ähnlichen Forschungsfragen der Zuschreibung befasst. ${ }^{6}$ Aber kurz nachdem wir die Arbeit aufgenommen hatten, wurde schnell deutlich, wie viel mehr dieses Projekt leisten konnte. Innerhalb weniger Monate hatte Sapp eine Suchmaschine entwickelt, mit der wir unsere gesamte musikalische Datenbank abfragen konnten und als Suchergebnis vollständige Partituren erhielten, in denen die Treffer hervorgehoben waren. Bald darauf entwickelte er eine ganze Reihe von Analysewerkzeugen: eine »piano roll«, die eine ausgewählte Partitur graphisch darstellt, indem jede Stimme durch eine spezifische Farbe repräsentiert wird; eine Histogrammanzeige der Stimmumfänge etc. All das führte zu einer konzeptionellen sowie praktischen Ausweitung des Projekts. Wenngleich wir uns weiterhin mit Problemen der Zuschreibung beschäftigten, interessierte uns nun eine größere Bandbreite an Forschungsfragen, die sich mit Beziehungen zwischen Stücken, Komponisten, Genres und sogar ganzer Epochen befassten: Wie verändert sich Stil im Laufe der Zeit? Was unterscheidet ein Lied von einer Messe? Was unterscheidet einen Komponisten von anderen?

Im Laufe der letzten Jahre haben wir das Projekt in verschiedene Richtungen erweitert: durch den Aufbau eines benutzerfreundlichen Webinterfaces, die Zusammenarbeit mit Informatikern und Elektrotechnikern an statistischen Modellen, die Entwicklung diverser Standards für die Kodierung der Musik ${ }^{7}$ und die Präsentation unserer Arbeit auf internationaler Ebene. Hierdurch haben wir erkannt, dass die Leistungsfähigkeit des JRP mit der zunehmenden Menge an Musik, die wir in die Datenbank aufnehmen, steigt, sodass sowohl stetig neues Repertoire zur Datenbank hinzugefügt als auch »Datenspenden« anderer Wissenschaftler angenommen werden. ${ }^{8}$ Derzeit stehen wir kurz davor, ein neues Formanalyse-Tool zu implementieren, das gemeinsam mit den Stanford Libraries entwickelt wurde, und suchen nach Alternativen, um unsere Partituren ins Internet zu stellen, was einen noch besseren Zugang zu den Details einzelner Stücke

6 Joshua Rifkin, »Problems of Authorship in Josquin: Some Impolitic Observations; with a Postscript on Absalon, fili mi«, in: Proceedings of the International Josquin Symposium Utrecht 1986, hrsg. von Willem Elders und Frits de Haen, Utrecht 1991, S. 45-52.

7 https://wiki.ccarh.org/wiki/Josquin_Project_encoding_standards. Die Vorgehensweise, mit der wir neue Stücke in das JRP einbinden, garantiert ein hohes Maß an Genauigkeit im Hinblick auf den Notentext. Allerdings kodieren wir keine abweichenden Lesarten, da dies die Erweiterung der Datenbank hemmen würde. Wenn das Projekt nicht deutlich besser finanziert wird, gibt es keine Möglichkeit, jede Lesart zu kodieren, ohne die anderen Ziele zu vernachlässigen. In jedem Falle sind die meisten Forschungsfragen, die über das JRP gestellt werden können, nicht auf die vollständige Berücksichtigung aller Varianten angewiesen, von denen die überwältigende Mehrheit nur in äußerst geringem Maße Abweichungen aufweist. In den Fällen, in denen es sinnvoll erscheint, alle überlieferten Lesarten eines Stückes zu Rate zu ziehen, kann fast immer auf moderne Ausgaben zurückgegriffen werden. Es sei ausdrücklich betont, dass das JRP als hilfreiches Tool und nicht als Ersatz für kritische Editionen intendiert ist.

8 Vgl. den unteren Teil der Website https://josquin.stanford.edu/about/. 
erlauben würde (z. B. die Möglichkeit, innerhalb eines Stückes zwischen verschiedenen Suchergebnissen in unterschiedlichen Tabs zu wechseln).

Unser Projekt hat anderen Forschungen aus den Digital Humanities viel zu verdanken. Das für unsere Arbeit wichtigste Vorbild ist das Princeton Josquin Project, das unter der Leitung von Lewis Lockwood und Arthur Mendel in den 1970er-Jahren große Fortschritte in den für uns interessanten Gebieten machte. ${ }^{9}$ Damals war das Hauptproblem ein technologisches: Das Princeton Project musste noch Lochkarten verwenden, die eine schnelle Dateneingabe und -analyse - gelinde gesagt - sehr erschwerten. Das Projekt und die daraus hervorgegangenen Forschungsergebnisse waren dennoch ein Erfolg, so auch ein Aufsatz von Mendel, der heute eine Grundlage für unsere Arbeit bildet, die nahtlos an seine Erkenntnisse anknüpft. ${ }^{10}$

\section{Drei praktische Anwendungsfelder}

Das JRP birgt das Potential, den wissenschaftlichen Diskurs, der um die Musik der Renaissance geführt wird, zu erweitern und unsere Forschungsergebnisse an höheren Standards zu messen. Im Rahmen dieses Aufsatzes soll an drei Beispielen gezeigt werden, wie das JRP genutzt wurde und in Zukunft genutzt werden könnte, um messbare wissenschaftliche Einblicke zu gewinnen.

\section{Quintparallelen sind schlecht}

Die Josquin-Forschung wird seit Generationen durch methodische Probleme erschwert: ${ }^{11}$ Wie soll man den kompositorischen Stil Josquins charakterisieren, dessen Schaffensüberlieferung von unsicheren und widersprüchlichen Zuschreibungen geprägt ist, dessen Biografie deutliche Lücken aufweist und selbst von dessen gesicherten Werken keine Chronologie erstellt werden kann, über dessen

9 Vgl. Arthur Mendel, »Some Preliminary Attempts at Computer-Assisted Style Analysis in Music«, in: Computers and the Humanities 4 (1969), S. 41-52; und ders., "Towards Objective Criteria for Establishing Chronology and Authenticity: What Help Can the Computer Give?«, in: Josquin des Prez: Proceedings of the International Josquin Festival-Conference Held at the Juilliard School at Lincoln Center in New York City, 21-25 June 1971, hrsg. von Bonnie J. Blackburn und Edward E. Lowinsky, London 1976, S. 297-308.

10 Arthur Mendel, »Towards Objective Criteria« (wie Anm. 9). Sean Gallagher ist eine weitere wesentliche methodische Inspiration, »Syntax and Style: Rhythmic Patterns in the Music of Ockeghem and His Contemporaries «, in: Johannes Ockeghem: Actes du Xle Colloque international d'études humanistes. Tours, 3-8 février, 1997, hrsg. von Philippe Vendrix, Paris 1998, S. 681-705.

11 Vgl. hierzu Jesse Rodin, »Josquin and Epistemology«, in: The Cambridge History of FifteenthCentury Music 2015, hrsg. von Anna Maria Busse Berger und Jesse Rodin, Cambridge 2015, S. 119-136. 
Persönlichkeit meistens posthume Anekdoten zweifelhafter Glaubwürdigkeit Auskunft geben und über dessen kompositorisches Umfeld noch immer große Kenntnislücken bestehen?

Angesichts dieser fundamentalen Probleme haben wir unsere Urteilsfähigkeit vielfach überschätzt. Häufig wird auf ungenaue Beschreibungen (z. B. der »vorantreibende« Kontrapunkt Josquins - ist sein Kontrapunkt wirklich vorantreibender als andere?) und pauschale Behauptungen (z. B. über die Tendenz des Komponisten, sich nicht zu wiederholen, was er tatsächlich aber häufig tut) zurückgegriffen, anstatt konkrete, vergleichs- und evidenzbasierte Beobachtungen anzustellen. Ein weiteres, kleineres Problem stellt die Neigung dar, sich auf kontrapunktische »Regeln« zu verlassen, die zwar von späteren Repertoires abgeleitet werden können, aber unsere Sicht auf die Musik des 15. und 16. Jahrhunderts verzerren. Vor allem die bloße Präsenz von parallelen, perfekten Intervallen - insbesondere von Quintparallelen - in einem Josquin zugeschriebenen Werk wird häufig als Beleg dafür angeführt, ihm dieses abzusprechen.

Das »Parallel«-Tool des JRP knüpft an das Princeton Josquin Project an, indem es mit diesem Pauschalurteil aufräumt und Nutzern alle Parallelen perfekter Intervalle in einem ausgewählten Repertorium anzeigt. ${ }^{12}$ Zusätzlich zu einer vollständigen Übersicht aller betreffenden Passagen werden Quintparallelen in Partiturauszügen rot, Oktav- und Primparallelen grün markiert. ${ }^{13}$ Selbst eine Suche innerhalb der fünfzig Werke, deren Zuschreibung zu Josquin als am gesichertsten gilt, weist ca. 170 Parallelen perfekter Intervalle auf, wie ein Beispiel aus der Missa Gaudeamus zeigt (Abb. 1).

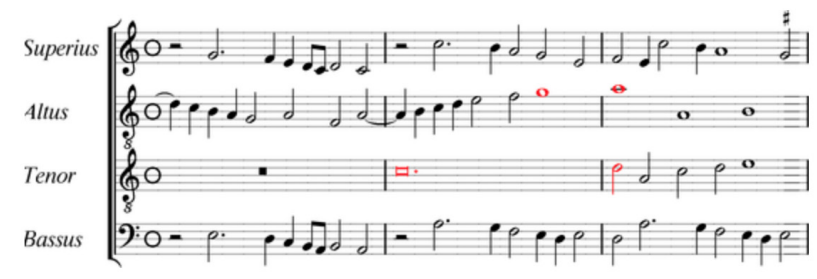

Abbildung 1. Beispiel eines Such-Ergebnisses mit dem »Parallek-Tool des Josquin Research Project (https://josquin.stanford.edu/analysis/parallel/).

Und dennoch (um Rifkin zu zitieren): »there are fifths and there are fifths. ${ }^{14}$ Daher unterscheidet das JRP zwischen sogenannten »harten« Parallelen (»hard

12 https://josquin.stanford.edu/analysis/.

13 Es ist von jeder Werkübersicht (z. B. https://josquin.stanford.edu/work/?id=Ock2004) aus möglich, eine vollständige Partitur zu laden, in der Quint- und Oktavparallelen hervorgehoben sind.

14 Private E-Mail-Korrespondenz (23. März 2017). 
parallel motion«), bei denen die parallelgeführten Noten gleichzeitig erklingen, und $»$ weichen« Parallelen, die erst im weiteren Stimmverlauf entstehen. ${ }^{15}$ Innerhalb dieser Kategorien kann noch feiner differenziert werden: Welche Stimmen sind für die Parallelbewegung verantwortlich? Wie viele Stimmen erklingen insgesamt? Treten die Quintparallelen in einer kadenzierenden Passage auf? Auf welche Notenwerte ist die Parallele geschrieben (d. h. erscheint sie »zufällig« im fließenden Stimmverlauf oder hat sie eine »strukturelle« Qualität)? Die Möglichkeit, jeden Fall mit anderen abzugleichen, erleichtert diese Art der Analyse, die dringend erforderlich ist, bevor Pauschalurteile gefällt oder gar Werke Josquin abgesprochen werden können.

Einerseits kann sicher festgestellt werden, dass Josquin gelegentlich perfekte Intervalle parallel führte, was angesichts des Umstandes, dass Komponisten seiner Generation keinen Anstoß an Quintparallelen nahmen, Sinn ergibt: Präziser formuliert, wandten sie diese »Regel« weniger streng an als Palestrina und dessen Zeitgenossen. ${ }^{16}$ Andererseits ist Josquin sehr bedacht darauf, wie und wann er Quintparallelen einfuihrt. Eine Quintparallele wird selbst in seinen »harten Parallelen dadurch abgemildert, dass die zweite Parallele in kurzen Notenwerten (d. h. Semiminimae oder Fusae) in einer oder in beiden Stimmen nur sehr beiläufig erklingen kann. In anderen Fällen ist die Parallele, ähnlich wie gelegentlich in Bachs Musik, durch das Hinzufügen von Akzidenzien zu einer kadenzierenden Wendung abgedämpft: Das erste Intervall wird zu einer verminderten statt einer reinen Quinte. Gerade im Vergleich mit anderen Komponisten oder mit Werken, die Josquin nicht eindeutig zugeschrieben werden, zeigt sich, dass er äußerst selten auffällige, »harte « Quintparallelen komponiert. ${ }^{17}$ Insgesamt zeigen diese Erkenntnisse, dass die bloße Präsenz von Quint- und Oktavparallelen kaum als Grundlage für Werkzuschreibungen dienen kann. Im Allgemeinen macht das »Parallel «Tool des JRP deutlich, dass weitere Untersuchungen zu diesem Thema nötig sind.

15 Vgl. https://josquin.stanford.edu/analysis/parallel/.

16 Obwohl das Verbot von Quint- und Oktavparallelen auf das 14. Jahrhundert zurückgeht, ist diese Regel in Josquins Ära in der theoretischen Literatur nicht so prominent wie sie es später einmal wurde. Vgl. Richard L. Crocker, »Discant, Counterpoint, and Harmony«, in: Journal of the American Musicological Society 15 (1962), S. 1-21, besonders S. 10-11; und Johannes Tinctoris, The Art of Counterpoint, übers. von Albert Seay, Rom 1961 (Musicological Studies and Documents, 5), S. 133-134.

17 Dennoch bleiben einige »schlechte« Parallelen. Vgl. z. B. (in grob chronologischer Folge) Missa L'homme armé sexti toni, Credo, T. 243; Missa Gaudeamus, Gloria, T. 32-33; Liber generationis, T. 124; und Missa Malbeur me bat, Credo, T. 116. Für den spektakulärsten Fall, vgl. Missa Faisant regretz, Credo, T. 122, in dem die Außenstimmen unverhohlen in Duodezimparallelen im homorhythmischen Satz möglicherweise eine Art Tonmalerei darstellen (der Text dazu lautet »unum baptisma«). 


\section{Edieren ist schwierig}

Jeder, der an wissenschaftlichen Editionen arbeitet, wird sich einmal gewünscht haben, dass ein bestimmtes Werk nur anhand einer einzigen Quelle überliefert ist. Auch wenn für die Editionsarbeit mehrere Textzeugen hilfreich sein können (und so frustrierend unica sein können), wird sie umso komplexer, wenn zwei, drei oder gar zwanzig Quellen die Grundlage bilden. Dieses Problem verschärft sich zusehends, je mehr Bedeutung der Textkritik beigemessen wird. Auch hier finden sich überall Problemfelder: Zu Repräsentationszwecken aufwendig gestaltete Manuskripte strahlen zwar Autorität aus, überliefern jedoch häufig eine schlechtere Lesart des Notentextes; eine Variante, die scheinbar zwei Quellen in Verbindung zueinander bringt, stattdessen aber wohl eher von verschiedenen Kopisten unabhängig voneinander eingebracht wurde; und offenkundige Eingriffe von genialen komponierenden Schreibern, die sich als zufällige Fehlerkorrekturen von heute nicht mehr erhaltenen Vorlagen erweisen. Diese Fälle werfen ein Schlaglicht auf Fragen, die beim Edieren gestellt werden müssen: Was ist die beste (meist heißt dies: die einfachste) Erklärung für die entdeckte Varianz? Welche Varianten sind bei der Festlegung des Stemmas wichtig und welche nicht? (Die meisten sind es nicht.) Und am wichtigsten: Welche musikalische Lesart diente als Vorlage für die anderen?

Bei der Edition von Josquins Missa L’homme armé sexti toni wünschte ich mir häufiger, sie sei ein Unikum, da die Quellen zu dieser Messe so kompliziert wie zahlreich sind: Teile des Werkes sind in 23 Quellen überliefert, darunter neun, die im Zeitraum von ca. 1498-1508 erstellt wurden. ${ }^{18}$ Obwohl diese Quellen sich leicht in zwei Hauptgruppen gliedern lassen, »italienische« und »nördliche«, hat keine der beiden eine offensichtliche Priorität, da in diesem Fall beiden eine gemeinsame Vorlage zu Grunde liegt. Daher besteht die angemessenste Lösung darin, beide Quellengruppen in einer Edition zusammenzuführen, wobei der Editor häufig von Fall zu Fall über die Quellenhierarchie entscheiden muss.

In einigen wenigen Passagen liegt die Antwort auf der Hand. Häufiger sind jedoch kreative Lösungen gefragt, um für die Bevorzugung einer bestimmten Quelle zu argumentieren. Hierzu ist es wichtig zu begründen, weshalb eine Quelle im Kontext des Werkes oder der generellen Gewohnheiten des Komponisten plausibler ist, wobei das JRP behilflich sein kann. Der Herausgeber kann sowohl nach melodischen und rhythmischen Varianten im Werk selbst als auch im gesamten Schaffen des Komponisten suchen. Dies ermöglicht Schlussfolgerungen zum Vorkommen bestimmter musikalischer Phänomene innerhalb eines Werks oder

18 Josquin des Prez, New Edition of the Collected Works (New Josquin Edition), 30 Bde., Amsterdam 1987-2015, Bd. 6, hrsg. von Jesse Rodin; und im beigefügten kritischen Kommentar S. 5-91, besonders S. 25-43. 
zu kompositorischen Eigenheiten von Josquin. Diese Folgerungen stellen keine endgültigen Beweise dar, sind aber evidenzbasiert und ermöglichen die größtmögliche Annäherung an eine fundierte Problemlösung.

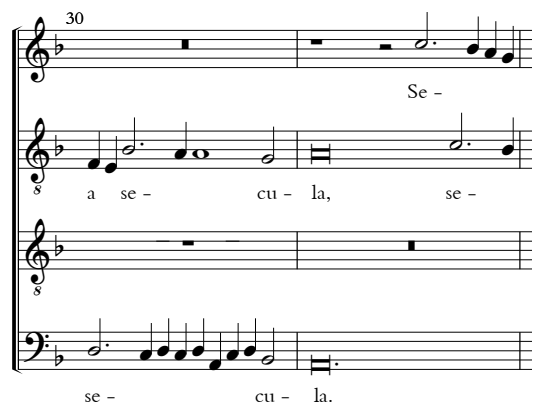

Italian

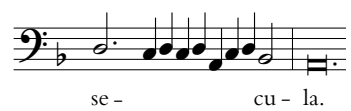

Notenbeispiel 1. "Italiensche" und "nördliche» Varianten (Josquin, Missa L'homme armé sexti toni, Credo, T. 30f., bassus)

Northern

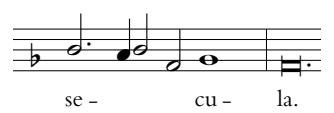

Eine besonders komplexe Passage findet sich im Credo (Notenbeispiel 1). In den nördlichen Quellen enthält es ein Duo, in dem der Bassus eine unauffällige Wechselnoten-Figur singt, die eine phrygische Kadenz nach $a$ antizipiert. Die Stimmführung ist in den italienischen Quellen hingegen deutlich lebendiger: Nachdem der Bassus auf einer punktierten halben Note $d$ angelangt ist, bewegt er sich dann in großen Notenwerten größtenteils zwischen $c$ und $d$. So singt er sieben aufeinanderfolgende Viertelnoten vor der abschließenden Kadenz. In keiner Version liegt ein kontrapunktischer Satzfehler oder ein Textierungsproblem vor. Daher muss zwischen zwei Standardinterpretationen entschieden werden: (a) Entweder schmückte der Kopist des »italienischen« Hyparchetyps Josquins Kadenzformel aus oder (b) ein »nördlicher« Kopist verschlankte Josquins exzentrische Roulade.

Die erste Interpretation scheint anfangs plausibler, da Schreiber häufig dazu tendieren, eher auszuschmücken als zu vereinfachen. Tatsächlich sind in den überlieferten italienischen Kopien im Vergleich zu den nördlichen mehr Verzierungen enthalten. Allerdings entspricht diese besondere Art der Ausgestaltung nicht geläufigeren Beispielen. Im Unterschied zu solchen Fällen, in denen der Schreiber einen Terzsprung mit einer Durchgangsnote auffüllt oder eine Kadenzformel mit einer Drehfigur aus zwei oder drei Noten verziert, weist die »raffinierte « Version hier eine viel aufwendigere Behandlung auf als eine minimale Ergänzung. Daher ist zu vermuten, dass die italienische Lesart eine lectio difficilior repräsentiert - eine »schwerere« und daher letztlich plausiblere Fassung. 
Zur Beantwortung dieser Frage muss noch eine weitere gestellt werden: Wie kann diese melodische Tonfolge sowohl innerhalb der Messe als auch in anderen Kompositionen, deren Zuschreibung zu Josquin gesichert ist, kontextualisiert werden? Ausgehend von der Werkübersicht des JRP für die Missa L’homme armé sexti toni ${ }^{19}$ können Bezugsmuster gesucht werden, indem die Suchbegriffe eingegeben werden:

Intervall: $-2+2-2+2$

Rhythmus: 2.444 eine Melodie, die sich schrittweise abwärts, aufwärts, abwärts, aufwärts bewegt

eine punktierte halbe Note gefolgt von drei Viertelnoten

Eine teilweise analoge Passage findet sich im Pleni (T. 33-34). ${ }^{20}$ Sowohl in der italienischen als auch in der nördlichen Überlieferung beginnt die untere Stimme des Duos eine kreisende Bewegung, die derjenigen im Credo sehr ähnelt. Somit lässt sich zumindest festhalten, dass keine außergewöhnlichen Abweichungen von Josquins melodischer Praxis als Basis für die Edition angenommen werden müssen, wenn man die italienische Lesart dieser Messe als Grundlage wählt.

Das JRP ermöglicht noch einen weiteren Schritt. Eine Suche nach diesen Mustern auf der zentralen Suchseite ${ }^{21}$ liefert eine weitere analoge Stelle eines Josquin gesichert zugeschriebenen Werks: das Benedictus der Missa Malheur me bat (T. 182), in dem wiederum die Kreisbewegung in der Unterstimme eines Duos erklingt, womit diese Analogie aber endet, da die betreffende Stimme schließlich auf ganz anderem Wege zur Kadenz geführt wird als der Bassus. Die Beziehung wird dennoch deutlich.

Tritt man noch einen weiteren Schritt zurück, so lässt sich beobachten, dass die italienische Überlieferungsvariante Josquins »obsessive compositional personality« abbildet, die ich an anderer Stelle beschrieben habe. ${ }^{22}$ Diese drückt sich u. a. in seiner Vorliebe für melodische, kreisende Bewegungen aus, die Kadenzen vorangestellt sind. Auch wenn diese Kompositionsweise kein Alleinstellungsmerkmal Josquins ist, so findet sich diese Figur doch sehr häufig in seinem Schaffen.

All dies beantwortet die Frage wie so oft nicht eindeutig. Es gibt keinen schlagenden Beweis - keine Passage in dieser Messe oder einem anderen Josquin gesichert zugeschriebenen Werk, in dem sich der gleiche Bassus in

19 https://josquin.stanford.edu/work/?id=Jos0602c.

20 Dieses Muster erscheint wieder im Gloria tua (T. 52-53), eine Passage, die abgesehen von der Transposition mit dem Pleni identisch ist.

21 https://josquin.stanford.edu/search/.

22 Jesse Rodin, »An Obsessive Compositional Personality«, in: Josquin's Rome. Hearing and Composing in the Sistine Chapel, New York 2012 (AMS Studies in Music), S. 41-97. 
seiner kadenzvorbereitenden Funktion findet. Darüber hinaus behält die nördliche Überlieferungsvariante, zu der sich sogar im Kyrie eine Analogie findet (T. 60-61), ihre Plausibilität. Auch wenn es schwer vorstellbar ist, dass ein Schreiber eine einfache Geste so frei ausgestaltet hat, leuchtet es ein, dass ein behutsam vorgehender Kopist die Stelle vereinfacht haben könnte; oder dass er sich (evtl. wahrscheinlicher) mit einem Fehler konfrontiert sah und die sinnvolle Lösung, die in der nördlichen Lesart überliefert ist, festhielt. ${ }^{23}$ Insgesamt spricht das Verhältnis von Wahrscheinlichkeiten in Bezug auf die Schreiberpraxis in Verbindung mit Josquins kompositorischen Neigungen hier für die italienische Lesart. Selbst wenn dies nicht mit absoluter Sicherheit festgestellt werden kann, so ermöglicht das JRP es dennoch, die eigenen Argumente und editorischen Entscheidungen auf ihre Stichhaltigkeit zu prüfen.

\section{Große Intervalle sind selten - und hilfreich bei Zuschreibungen}

Es herrscht Konsens darüber, dass der bedeutendste stilistische Wandel der frankoflämischen Polyphonie des 15. Jahrhunderts um 1480 im Zuge eines Generationenwechsels von Ockeghem, Antoine Busnoys und Johannes Regis zu Josquin, Jacob Obrecht, Heinrich Isaac und La Rue erfolgte. ${ }^{24}$ Dieser Wandel wird meist mit dem Aufkommen der Imitation, dem Niedergang der formes-fixes Chanson und einer stärkeren Tendenz zur syllabischen Textverteilung erklärt. Die musikalischen Grundelemente und subtilen Veränderungen in der kompositorischen Praxis, die Ockeghem so deutlich von Josquin unterscheiden, sind aber erst oberflächlich untersucht. Eine gründliche Kenntnis der Musik ist eine Vorbedingung, um diesen Wandel beobachten und interpretieren zu können. Dieses Wissen, das durch Hören, Singen und Lesen der Partitur gewonnen wurde, reicht in manchen Fällen jedoch nicht aus, sodass man hier auf »a large mass of facts $\ll^{25}$ zurückgreifen muss, wie Franco Moretti es formuliert hat. In Ermangelung eines Noten-zählenden Heeres braucht es hierfür digitale Werkzeuge.

23 Man beachte die $c$-d-Semiminimae, die drei Mal in der italienischen Version erscheinen (Bsp. 2). Der Kopist des nördlichen Hyparchetyps könnte versehentlich zum dritten Tonwechsel $c-d$ gesprungen sein und dabei $c-d-c-d-A-c$ ausgelassen haben; die uns überlieferte nördliche Lesart könnte eine plausible Korrektur sein. Im Gegensatz dazu ist es nur schwer vorstellbar, wie die versehentliche Eintragung der zwei Minimae in der nördlichen Version - oder jedes anderen analogen Kopierfehlers - eines Kopisten als Grundlage für das Entstehen der italienischen Lesart gedient haben könnte.

24 Eine frühere Fassung einiger dieser Inhalte habe ich bereits 2012 auf der Tagung der American Musicological Society (New Orleans) mit Clare Bokulich präsentiert. Der Beitrag hieß »A Large Mass of Facts«.

25 Franco Moretti, Graphs, Maps, Trees: Abstract Models for a Literary History, London und New York 2005, S. 3. 
Vor allem die Tonhöhe ist ein Parameter, der gerade in Bezug auf die Setzweise einfacher melodischer Gesten systematisch erforscht werden muss. Es ist bekannt, dass Ockeghem und seine Zeitgenossen gerne lange melodische Linien komponierten, die schnell einen großen Stimmumfang durchschritten, was die Sänger an ihre Grenzen führte. Ebenso klar ist, dass Josquin und die Komponisten seiner Generation im Gegensatz dazu vermehrt kurze Motive nutzten, die imitiert, sequenziert und mit einem Ostinato versehen werden konnten. Dies wirft die Frage auf, welche dieser beiden Strategien in der melodischen Gestaltung vermehrt auf Tonsprünge zurückgreifen. Die Beantwortung dieser Frage ist sehr vielschichtig (vgl. Tabelle 1 und Abb. 2; in Abb. 2 nutzt jeder Graph einen anderen Maßstab). ${ }^{26}$

Trotz Ockeghems Reputation, melodisch, rhythmisch und auf andere Weise komplex zu schreiben, ist er in Bezug auf große Tonsprünge der konservativste Komponist der oben genannten. ${ }^{27}$ Dies mag auf den ersten Blick überraschen, erscheint bei näherem Betrachten aber verständlich: Je heterogener ein kompositorischer Parameter ist, was Carl Dahlhaus »materiale Differenzierung « nennt, desto strenger wird mit benachbarten Parametern umgegangen. ${ }^{28}$ Ein paar Beispiele sollen dies verdeutlichen: Rhythmische Komplexität findet sich in Messen des 15. Jahrhunderts nicht im vollen, vierstimmigen Satz, sondern in zweistimmigen Passagen; explizite, >färbende $<m i$ und $f a$ Zeichen (d. h. Kreuz- und B-Vorzeichen) treten nicht so sehr in dichten polyphonen Passagen auf, sondern häufiger in homorhythmischen Kontexten; und schließlich verzichtet Ockeghem auf häufige, akrobatische Tonsprünge zugunsten der Kultivierung seiner Komplexität. In dieser Hinsicht ist es interessant, dass die Komponistengeneration, die sich um 1480 etablieren konnte, wieder Anschluss an Du Fay suchte und dass Busnoys, der jetzt als eine Art »Übergangsfigur« gesehen wird und einige der stilistischen Entwicklungen des späten 15. Jahrhunderts initiierte, auch bezüglich großer Tonsprünge eine »Übergangsfunktion $\ll$ inne hatte. ${ }^{29}$

26 Vgl. auch Cory McKay, Julie Cumming und Ichiro Fujinaga, »Characterizing Composers Using jSymbolic2 Features«, in: Extended Abstracts for the Late-Breaking Demo Session of the 18th International Society for Music Information Retrieval Conference, Suzhou, China, 2017, https://ismir2017.ismir.net/.

27 Im Durchschnitt nutzt La Rue Oktavsprünge etwas seltener als Ockeghem, allerdings verwendet er Sextsprünge mehr als doppelt so häufig.

28 Dieser Punkt ist eng mit Dahlhaus' Unterscheidung von »materialer« und »funktionaler « Differenzierung verknüpft; vgl. Analysis and Value Judgment, trans. Siegmund Levarie, New York 1983, S. 43-45, ursprünglich erschienen als: Analyse und Werturteil, Mainz 1970 (Musikpädagogik. Forschung und Lehre 8), S. 50-54. Ich möchte Sean Gallagher für den persönlichen Austausch seiner Ideen zu diesem Thema danken.

29 Es ist erstaunlich, dass Quintsprünge sich als weniger ergiebig erweisen. Fünf oder sechs der hier behandelten Komponisten komponieren zwischen 26.3 (gesichert Josquin) und 30.3 (Du Fay) Quintsprünge pro 1000 Noten - eine enge Verteilung. De Orto schreibt im Durchschnitt 35.7 Quintsprünge pro 1000 Noten. 

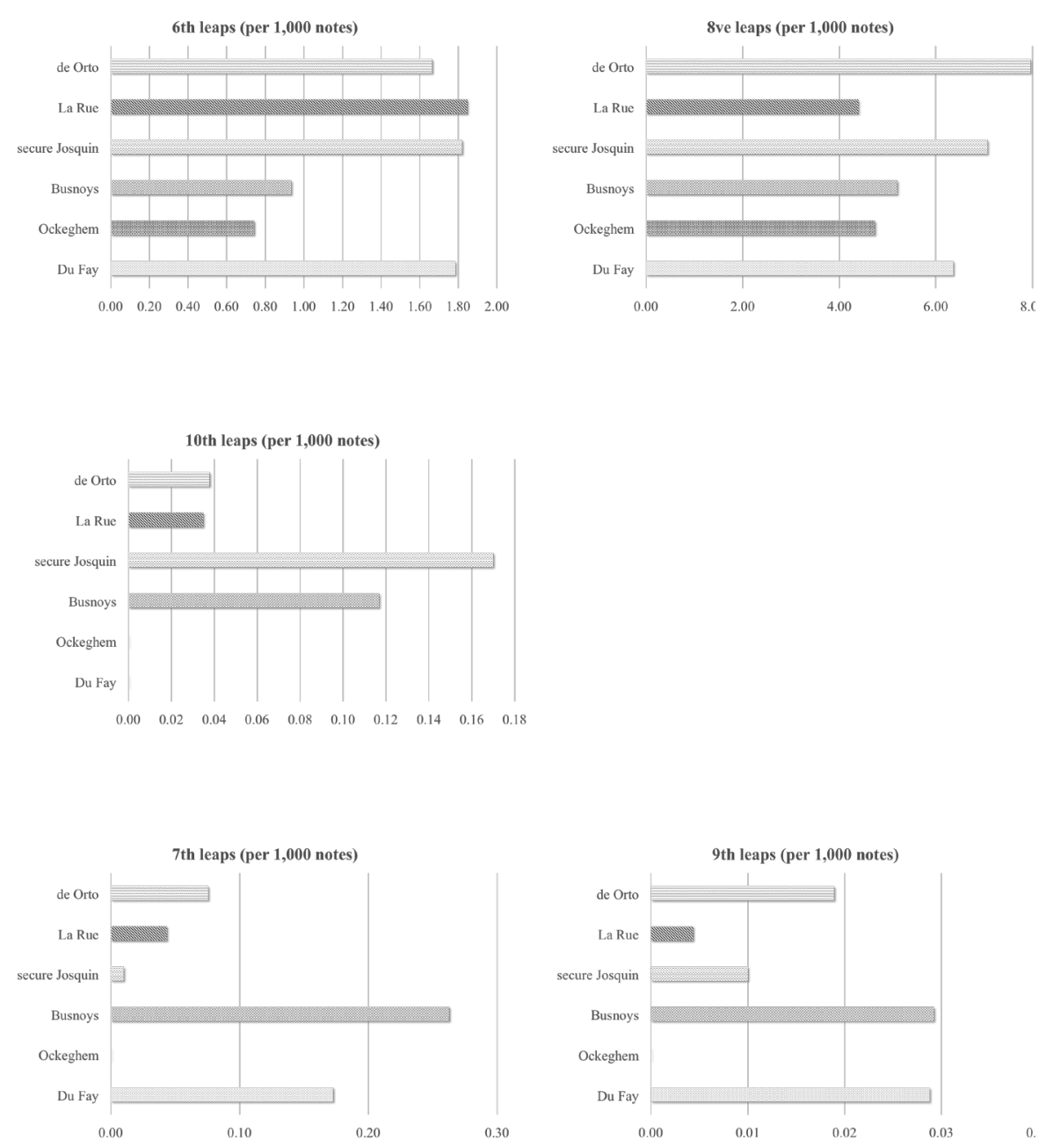

Abbildung 2. Große Sprünge in der Musik einiger Komponisten des 15. Jahrhunderts.

Was Oktavsprünge angeht, so ähnelt La Rues Vorgehensweise der Ockeghems, wodurch sie als deutlich konservativer einzuordnen ist als die Praxis seiner Zeitgenossen. Im Kontext seiner Messen bedeutet dies, dass im Durchschnitt vier Oktavsprünge pro Messensatz zu erwarten sind im Vergleich zu sieben oder acht in den Kompositionen Marbrianus de Ortos und Josquins. Je nach Blickwinkel mag dieser Unterschied gering erscheinen. Allerdings darf davon ausgegangen werden, dass diese Differenz signifikant ist, da sie auf einem Datensatz von über 500.000 Noten (knapp der Hälfte aller Noten, die das JRP insgesamt umfasst) 
basiert. Die große Anzahl an Beispielen - selbst Ockeghems überlieferte Werke enthalten mehr als 400 Oktavsprünge - erfordert weitere Untersuchungen.

Im nochmaligen Rückgriff auf die grobe Einteilung nach Generationen (siehe Tabelle 1 und Abb. 2) zeigt sich, dass diese Aufteilung auch auf die größten Intervallsprünge im Repertorium angewendet werden kann. Es existieren nahezu keine Dezimsprünge in der Musik Ockeghems oder Du Fays. Während dies durchaus auch für die Musik de Ortos und La Rues gilt, ist Josquin (bislang) der einzige Komponist, in dessen Werken sich gleich mehrere Beispiele für Tonsprünge dieser Art aufzeigen lassen. ${ }^{30}$ Diese Dezimsprünge treten besonders in kirchenmusikalischen Kompositionen zu vier oder mehr Stimmen gehäuft auf und finden sich meist im Altus und Bassus. Sie sind mit zwei Ausnahmen immer aufsteigend und knapp drei Viertel dieser Sprünge sind auf kurze Notenwerte (Minima oder gar Semiminima) gesetzt. Manchmal erscheinen sie in Form einer repetitiven Geste, wie die drei dicht aufeinanderfolgenden Dezimen aus Preter rerum seriem verdeutlichen (vgl. Notenbeispiel 2). In dieser Passage katapultieren sich die beiden Bassus-Stimmen sukzessive in eine mittlere Stimmlage. Vergleichbar mit etwa der Hälfte aller Dezimsprünge Josquins münden auch diese Anstiege jeweils in eine Kadenz, die eine Terz unterhalb des höchsten Tones liegt und deren Intensität (vermutlich) durch die schneidende Dissonanz von fis und $f^{\prime}$ gesteigert wird. Tatsächlich erreicht diese Musik gegen Ende der secunda pars ihren Höhepunkt, nach welchem die dichte Polyphonie, innerhalb derer die Dezimsprünge auftreten, plötzlich von einem deutlich leichteren Dreiermetrum abgelöst wird.

Anhand dieser Passage wie auch an anderen ähnlichen Stellen zeigt sich, dass Josquin sich eine Art melodische Athletik zu Nutze machte, die andere Komponisten des 15. Jahrhunderts meist vermieden. Diese durch größere Intervallsprünge gekennzeichnete Stimmführung hat allerdings zur Folge, dass dem Komponisten nur eingeschränkte kompositorische Möglichkeiten nach Oktav- oder Dezimsprüngen verbleiben. Ein Komponist wie Ockeghem hätte Sprünge dieser Art womöglich vermieden, um sich nicht von solchen Konventionen einschränken zu lassen: So zeigt sich wiederum, dass sich eine heterogene Behandlung der Melodik auf benachbarte musikalische Parameter begrenzend auswirkt.

Die Untersuchung von Septimsprüngen erweist sich als unkomplizierter, da kaum ein Komponist dieser Zeit sie schreibt. Du Fay und de Orto konfrontieren Sänger nur selten mit dieser ungewöhnlichen melodischen Wendung; noch seltener findet sie sich bei La Rue, Ockeghem und zuletzt bei Josquin, wenn man

30 Im gesamten vom JRP kuratierten Repertorium finden sich nur zwei größere Sprünge: Dies sind zwei Duodezimsprünge in der Vertonung von O Venus Bant, die sowohl Josquin als auch Gaspar zugeschrieben wird (NJE 27.29). Wie zu vermuten war, erscheinen beide Sprünge im Contratenor. 


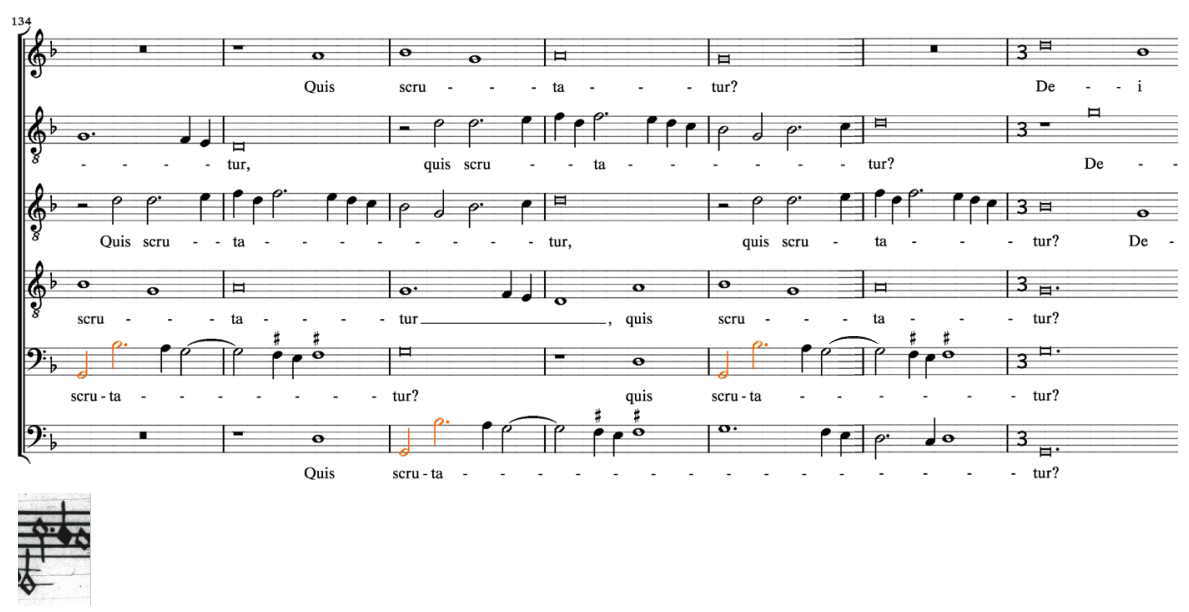

Notenbeispiel 2. Dezim-Sprünge in Josquin's Preter rerum seriem, T. 134-140.

zunächst nur die ihm gesichert zugeschriebenen Werken in den Blick nimmt. ${ }^{31}$ Die einzige Ausnahme bildet in diesem Zusammenhang bislang Busnoys, der Septimsprünge vergleichsweise häufig komponiert; hier treten sie vor allem im Contratenor seiner Chansons auf, einem Stimmtypus, der besonders durch Tonsprünge charakterisiert ist. ${ }^{32}$ Glücklicherweise hilft diese Erkenntnis bei der Klärung eines Zuschreibungsproblems: Das berühmte Lied Quand ce viendra, das Busnoys im Laborde- und Dijon-Chansonnier zugeschrieben ist, aber »hockengem « in Escorial B, weist zwei Septimsprünge auf, die jeweils im Contratenor auftreten. ${ }^{33}$ In Anbetracht dessen, dass keiner der vier Septimsprünge Ockeghems in der Contratenor-Stimme einer Chanson zu finden ist, darf die Zuschreibung von Quand ce viendra zu Busnoys als gesichert gelten.

Noch seltener als Septimen sind Nonensprünge, für die sich eines der außergewöhnlichsten Beispiele unter den gesicherten Zuschreibungen Josquins gegen Ende des Gloria aus der Missa Malheur me bat kurz vor dem Wiedereinsatz des klimaktischen Materials findet, das den Messensatz zum Ende führt. ${ }^{34}$ In T. 144 springt die Altus-Stimme auf ihre höchste Note $\left(a^{\prime}\right)$, um danach das hohe $g$ ' drei Takte lang zu halten (T. 145-47). Der Superius kehrt wiederholt auf seine höchste

31 Die zwei Fälle, in denen Septimsprünge doch nachweisbar sind, finden sich im Miserere mei deus, T. 218, Tenor 2 und im Virgo salutiferi/Ave Maria, T. 78, Bassus.

32 Diese Gebrauchsweise findet sich in acht von insgesamt neun Beispielen; der letzte tritt im Superius der Missa L'homme armé auf.

$33 \mathrm{Zu}$ den Quellen vgl. David Fallows, A Catalogue of Polyphonic Songs 1415-1480, Oxford 1999, S. 335-336.

34 Für eine Edition vgl. https://josquin.stanford.edu/cgi-bin/jrp?a=notationEditText\&f=Jos090lb. 
Note $(e ")$ in Dezimparallelen mit dem Bassus zurück, bevor er zur abschließenden Kadenz absteigt (T. 150). In diesem Kontext erweist sich der Altus-Sprung als klimaktisch, gar als triumphierend, indem der Altus den affektiven Höhepunkt des Satzes einleitet. Die Passage verdeutlicht den überwältigenden Effekt, den diese außergewöhnliche Kompositionsweise hat. ${ }^{35}$

Auf dieser Grundlage ist es möglich, das JRP zu nutzen, um ein weiteres, komplizierteres Zuschreibungsproblem anzusprechen: Die Missa Une musque de Biscaye, ein Stück, das möglicherweise mit Josquins Chanson gleichen Namens verwechselt worden ist. ${ }^{36}$ Wie Rob Wegman angemerkt hat, ist diese Messe in mehreren Quellen überliefert, von denen jede eine Zuschreibung zu Josquin erhält; darüber hinaus zählt die früheste von ihnen (Berlin 40021, ca. 1495-97 kopiert) zu den ältesten überlieferten Quellen von Josquins Messvertonungen. ${ }^{37}$ Dennoch haben Jaap van Benthem und Rifkin festgestellt, dass all diese Quellen eine Vielzahl von Fehlern verbindet, sodass die mehrfachen Zuschreibungen zu Josquin sich effektiv auf eine einzige reduzieren lassen. ${ }^{38}$ Für sich genommen bildet dies kein Argument gegen Josquins Autorschaft, zumal das relativ frühe Datum von Berlin 40021 ein gewisses Maß an Autorität auszustrahlen scheint..$^{39}$ Dennoch mahnen mehrere Überlegungen zur Vorsicht, darunter die geographische Entfernung des Manuskripts von Josquin (vermutlich wurde es in Süddeuts chland kopiert), das Fehlen mehrerer Überlieferungen mit Zuschreibung, eine Reihe stilistischer Ungereimtheiten im Vergleich zum Korpus der gesichertsten Werke ${ }^{40}$ Josquins sowie die Möglichkeit einer Verwechslung mit der Josquin gesichert zugeschriebenen Chanson Une musque de Biscaye. ${ }^{41}$

35 Der einzige andere gesichert zugeschriebene Fall findet sich in Vultum tuum, Altus, T. 428-429, in dem große Notenwerte (Brevis - Brevis) den besonderen Charakter des Sprungs dämpfen.

36 Die folgende Analyse setzt den Ansatz zweier früherer computerbasierten Untersuchungen fort: Mendel, »Towards Objective Criteria« (wie Anm. 9), S. 300-302; sowie Anne-Emmanuelle Ceulemans, »A Stylistic Investigation of Missa Une mousse de Biscaye, in the Light of Its Attribution to Josquin des Prez«, in: Tijdschrift van de Koninklijke Vereniging voor Nederlandse Muziekgeschiedenis 48 (1998), S. 30-50.

37 Rob C. Wegman, »Who Was Josquin?«, in: The Josquin Companion, hrsg. von Richard Sherr, New York und Oxford 2000, S. 21-50, besonders S. 30-33.

38 Jaap van Benthem, »Was >Une mousse de Biscaye< Really Appreciated by Lami Baudichon?«, in: Muziek \& Wetenschap 4 (1991), S. 175-94; und Joshua Rifkin, »Masses and Evidence: Petrucci’s Josquin«, unveröffentlichter Aufsatz. Vgl. auch Bonnie J. Blackburn, »Masses Based on Popular Songs and Solmization Syllables«, in: The Josquin Companion (wie Anm. 37), S. 51-87, hier: S. 72-76.

39 In seinem 2010 für die New Josquin Edition erschienenen Band verteidigt Martin Just die Autorschaft Josquins (Bd. 5, kritischer Bericht, S. 79-89). Vgl. auch die dort zitierte zusätzliche Forschungsliteratur.

40 Vgl. Wegmans angebrachte methodische Kritik von van Benthems komparativer Analyse in »Who Was Josquin?« (wie Anm. 37).

41 Justs Vorschlag (New Josquin Edition, Bd. 5, kritischer Kommentar, S. 82), dass Werke mit ähnlichen Titeln unterschiedlicher Genres nicht verwechselt wurden, wird durch Kompositionen wie die Missa Missus est angelus widerlegt, die auf Josquins vierstimmiger Motette basiert und sowohl ihm als auch Moulu zugeschrieben wird. 
In der Messe findet sich ein seltener Nonensprung im Kyrie/Agnus Dei (T. 83-84, b-c").42 Dieser Sprung ist nicht nur auf kurze Notenwerte komponiert (Minima - punktierte Minima), sondern erscheint auch in der prominentesten Stimme, dem Superius. Die zwei bereits erwähnten Nonensprünge aus den Josquin gesichert zugeschriebenen Werken treten im Altus auf und in großen bzw. relativ großen Notenwerten, sodass dies eine ganz andere musikalische Situation konstituiert. Selbst wenn die Suche auf alle 300 Werke Josquins ausgedehnt wird, die das JRP derzeit aufgenommen hat (und deren Zuschreibung zu Josquin in vielen Fällen zweifelhaft erscheint), kann nur ein weiterer Nonensprung gefunden werden. Dieser tritt tatsächlich im Superius in entsprechenden Notenwerten (Semibrevis - Minima) in der Missa Sub tumm presidium auf, die nahezu sicher von La Rue komponiert wurde.43 Einerseits erscheint es beunruhigend, dass sich eine solch charakteristische Geste in einem Werk eines vermutlich anderen Komponisten findet. Andererseits reicht dieser eine Sprung auf Grund der außergewöhnlichen Seltenheit von Nonensprüngen nicht aus, als Beweis gegen Josquins Autorschaft herangezogen zu werden. Als stilistische »Fingerabdrücke« erweisen sich Nonensprünge somit nicht als sonderlich hilfreich; ihre exotische Qualität (derzeit enthält das JRP insgesamt nur neun Beispiele und damit einem Anteil von unter eins von 100.000) deutet darauf hin, dass Komponisten sie bewusst einsetzten. Statistiken können dazu beitragen, musikalische Erscheinungsformen innerhalb eines bestimmten Werkes aufzuzeigen, die nicht dem einem Komponisten zugeschriebenen Stil entsprechen. Solche Merkmale gewinnen an Bedeutung, wenn sie aller Wahrscheinlichkeit nach unbewusst eingesetzt wurden.

Bedeutsamer sind daher die vier Septimsprünge in dieser Messe: dies entspricht einem Sprung unter 1500 Noten, d.h. im Durchschnitt fünfzig Mal häufiger als im gesichert zugeschriebenen Korpus. ${ }^{44}$ Alle vier Beispiele treten im Bassus mit der Tonfolge $G$ zu $f$ auf. Wenn man das Blickfeld auf das gesamte Repertorium des JRP erweitert, sind die Ergebnisse verblüffend. Trotz der relativen Seltenheit von Septimsprüngen im Vergleich zu häufigeren Intervallen sind sie mit knapp 100 Fällen in der gesamten Datenbank vergleichsweise häufig vertreten,

42 In dieser Messe ist das Agnus Dei mit dem Kyrie (»Agnus super Kyrie«) identisch.

43 Gloria, T. 53-54. Zu der Messe liegen fünf Zuschreibungen zu La Rue vor, nur eine Quelle von 1539 schreibt sie Josquin zu. Um dies zu kontextualisieren, sei darauf hingewiesen, dass es im gesamten Repertorium, das zurzeit im JRP aufgenommen ist, neun Nonensprünge gibt. Die übrigen Beispiele finden sich bei Agricola, Missa Malheur me bat, Sanctus, T. 36, Bassus; Busnoys, Quelque pauvre homme II, T. 48-49, Contratenor; Gaspar, Ave regina celorum mater, Motette V im Quam pulchra es-Zyklus, T. 58-59, Altus; Du Fay, Resvellies vous, T. 52-53, Contratenor; und de Orto, D'ung aultre amer, T. 12-13, Bassus. In den ersten drei Passagen ist wenigstens eine der zwei Noten des Tonsprungs eine Minima.

44 Diese treten auf im Gloria, T. 4 und 44-45, im Credo, T. 121 und im Sanctus, T. 157-58; bis auf den letzten Fall weisen sie kurze Notenwerte auf. Die gesamte Messe besteht aus 6.154 Noten. 


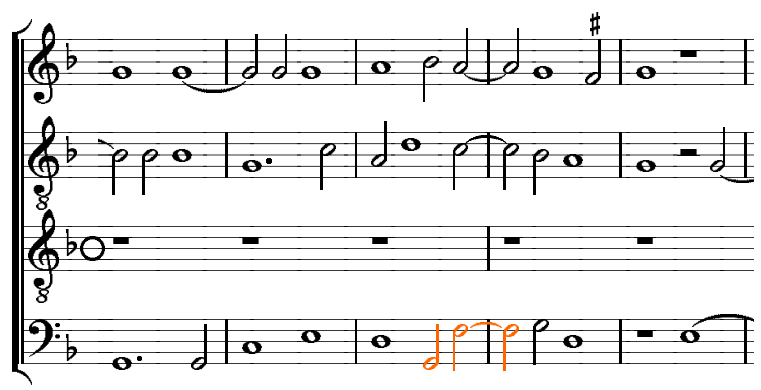

Notenbeispiel 3. Sept-Sprung in der Missa Une Musque de Biscaye (T. 121).

womit sie bei Zuschreibungsproblemen helfen können. ${ }^{45}$ Septimsprünge folgen grundsätzlich dem Modell der Missa Une musque de Biscaye: ein Anstieg des Bassus von der Finalis zur Septime darüber. Die untere Note erklingt meist auf dem Schlag, worauf eine Kadenz zur Finalis folgt (vgl. Notenbeispiel 3 von einem der zwei Beispiele aus der Messe). Dennoch lassen sich diese Tendenzen nicht in den Werken feststellen, die Josquin gesichert zugeschrieben sind und stattdessen den Septimsprung nutzen, um motivische Wiederholung oder eine fuga zu ermöglichen. Selbst wenn der »Josquin«-Korpus auf die Werke erweitert wird, die ihm weniger sicher zugeschrieben werden, bleibt die Korrelation zwischen Septimen mit melodischem Gehalt und motivischer Repetition bestehen, wohingegen sich zur Une musque-Messe keine Analogien finden. ${ }^{46}$ Ist es möglich, dass Josquin in nur einem Stück mehr Septimsprünge als üblich komponierte und jedes Mal eine konventionelle Form wählte, die er andernfalls vermied? Auch wenn dies theoretisch denkbar ist, so ist dies nicht wahrscheinlich.

Es gibt somit einen weiteren Grund, Josquins Autorschaft der Missa Una musque de Biscaye anzuzweifeln. In einem Fall wie diesem sprechen die Argumente, das Werk Josquin abzusprechen, für sich; es ist nicht nötig, einen Autor zu identifizieren, der mit größerer Wahrscheinlichkeit die Messe komponierte. Digitale Tools können nicht dabei helfen, eine solche Identifizierung mit Sicher-

45 Diese Praxis habe ich bereits für die Musik Busnoys' untersucht; eine signifikante Anzahl weiterer Beispiele könnte hier angeführt werden. Vgl. u. a. Anonymus, Donne vidue/Tarrach barach/ Jeus diray, T. 77; Du Fay, Craindre vous vueil, T. 24 und Adieu ces bons vins de Lannoy, T. 18-19; Gaspar, Tota pulchra es (Quam pulchra es-Zyklus VIII), T. 26; Jean Japart, Trois filles estoient, T. 20; de Orto, D'ung aultre amer, T. 30 (in zwei Stimmen); sowie die im Folgenden erwähnten.

46 Vgl. vor allem die Septimsprünge, die Wiederholungen ermöglichen, in Vous l'arez, T. 43-44, Bassus; und Si j'ay perdu (NJE 28.32), T. 42, Bassus. Bezeichnenderweise schwindet die Verbindung von Septimsprüngen und motivischer Repetition, wenn die Suche auf Werke ausgedehnt wird, deren Zuschreibung nicht gesichert ist. In jedem Fall enthält keines dieser Stücke so viele Septimsprünge wie die Une musque-Messe. 
heit vorzunehmen: Derzeit verfügt das JRP nicht einmal über die Mehrzahl der Werke von Isaac, Loyset Compere, Alexander Agricola oder Obrecht, um nur die vier prominentesten Zeitgenossen Josquins zu nennen. Allerdings habe ich an anderer Stelle über einen Komponisten geschrieben, der eine außergewöhnliche Vorliebe für Septimsprünge der Art aufweist, wie wir sie hier finden: Gaspar van Weerbeke. ${ }^{47}$ Wie bei den zuvor genannten Komponisten umfasst das JRP im Moment nur wenige Werke von Gaspar, ${ }^{48}$ was die weitere Verfolgung dieser Hypothese zu diesem Zeitpunkt erschwert. Mit einem stetig wachsenden Korpus könnte es einmal möglich sein, weitere Belege für van Benthems Vorschlag zu sam meln, dass er der Komponist sein könnte, oder die Zweifel zu bestätigen, die die Herausgeber der Gaspar-Ausgabe geäußert haben. ${ }^{49}$ Ein größerer Korpus wird es über Zuschreibungsfragen hinaus ermöglichen, weitere musikalische Phänomene zu untersuchen, die ihrerseits als überzeugende stilistische Merkmale identifiziert werden können.

In diesen Beispielen erweist sich das JRP als ein hilfreiches Tool, aber nicht als Wunderwaffe. Es ist wichtig zu beachten, dass diese Ressourcen falsch verwendet werden können, indem individuelle Beispiele nicht gründlich recherchiert, Daten falsch ausgelegt und Probleme des Textes, Genres und der Chronologie ignoriert werden. Um noch größere Fehler als die anfänglich beschriebenen zu vermeiden, sollten digitale Tools nie als Ersatz dafür dienen, Musik auf die »altmodische« Art durch Aufführungen, Hören, Partiturstudium und genaue Beschäftigung mit den Originalquellen und kritischen Ausgaben kennenzulernen. Im Hinblick auf ein Stück, das man nahezu auswendig kennt, gibt es dennoch immer neue Bereiche, die durch neue analytische Fragestellungen eröffnet werden können. Selbst wenn man hunderte Stücke sehr genau studiert hat, kann es hilfreich sein, ein Analysewerkzeug zu verwenden, das eine viel größere kompositorische Landschaft erschließt. Eine solche Möglichkeit bieten Ressourcen wie das JRP: Sie können Normen von Ausnahmen unterscheiden und Fragen beantworten, die sonst Monate aufwendiger Arbeit erfordern würden. Sie können sogar dazu beitragen,

47 Neun analoge Beispiele sind in Jesse Rodin, Josquin's Rome: Hearing and Composing in the Sistine Chapel, New York und Oxford 2012, S. 141-146, besonders in Tabelle 4.2 auf S. 142 zitiert.

48 Selbst unter diesen findet sich ein Nonensprung in entsprechenden Notenwerten, allerdings im Altus: https://josquin.stanford.edu/cgi-bin/jrp?a =notationWithEditWithText\&f=Gas2017e\&th $\mathrm{emax}=-\mathrm{I} \% 229 \% 22$, T. $58-59$.

49 van Benthem, »Was >Une mousse de Biscaye< Really Appreciated« (wie Anm. 38). Vgl. Eric F. Fiedler, »A New Mass by Gaspar van Weerbeke? Thoughts on Comparative Analysis«, in: Studien zur Musikgeschichte: Eine Festschrift fïr Ludwig Finscher, hrsg. von Annegrit Laubenthal und Kara Kusan-Windweh, Kassel 1995, S. 72-87. Andrea Lindmayr-Brandl und Paul Kolb diskutierten freundlicherweise ihre Zweifel an Gaspars Autorschaft mit mir (persönliche Kommunikation). 
Jesse Rodin

Fragen zu beantworten, von deren Existenz wir gar nicht wussten, indem sie auf ein musikalisches Charakteristikum oder eine Verbindung aufmerksam machen, die sonst verborgen geblieben wäre. Schließlich geht es nicht darum, Technologien um ihrer selbst willen zu verherrlichen, wie es zur Zeit Mode zu sein scheint, sondern sie in der Aufarbeitung der musikalischen Vergangenheit mit Bedacht einzusetzen. 


\section{APPENDIX}

Tabelle 1

Guillaume Du Fay (as of spring 2018, the JRP curates 34,742 notes by Du Fay)

\begin{tabular}{lcc} 
Intervall & Anzahl pro Tausend Noten & Anzahl der Sprünge \\
\hline Sexte & 1,78 & 62 \\
Septime & 0,17 & 6 \\
Oktave & 6,36 & 221 \\
None & 0,3 & 1 \\
Dezime & 0 & 0
\end{tabular}

Johannes Ockeghem (87,640 notes)

\begin{tabular}{lcc} 
Intervall & Anzahl pro Tausend Noten & Anzahl der Sprünge \\
\hline Sexte & 0,74 & 65 \\
Septime & 0,05 & 4 \\
Oktave & 4,74 & 415 \\
None & 0 & 0 \\
Dezime & 0,1 & 1
\end{tabular}

Antoine Busnoys (34,233 notes)

\begin{tabular}{lcc} 
Intervall & Anzahl pro Tausend Noten & Anzahl der Sprünge \\
\hline Sexte & 0,93 & 32 \\
Septime & 0,26 & 9 \\
Oktave & 5,2 & 178 \\
None & 0,03 & 1 \\
Dezime & 0,12 & 4
\end{tabular}

secure Josquin ( 153,551 notes $)$

\begin{tabular}{lcc} 
Intervall & Anzahl pro Tausend Noten & Anzahl der Sprünge \\
\hline Sexte & 1,82 & 279 \\
Septime & 0,01 & 2 \\
Oktave & 7,07 & 1086 \\
None & 0,01 & 2 \\
Dezime & 0,17 & 26
\end{tabular}


Jesse Rodin

Pierre de la Rue (229,629 notes)

\begin{tabular}{lcc} 
Intervall & Anzahl pro Tausend Noten & Anzahl der Sprünge \\
\hline Sexte & 1,85 & 424 \\
Septime & 0,04 & 10 \\
Oktave & 4,39 & 1009 \\
None & 0,004 & 1 \\
Dezime & 0,03 & 8
\end{tabular}

Marbrianus de Orto (52,862 notes)

\begin{tabular}{lcc} 
Intervall & Anzahl pro Tausend Noten & Anzahl der Sprünge \\
\hline Sexte & 1,66 & 88 \\
Septime & 0,15 & 4 \\
Oktave & 7,96 & 421 \\
None & 0,01 & 1 \\
Dezime & 0,04 & 2
\end{tabular}

